\title{
AVALIAÇÃO DE COMPLICAÇÕES RELACIONADAS À CPRE EM PACIENTES COM SUSPEITA DE COLEDOCOLITÍASE
}

\author{
EVALUATION OF ERCP-RELATED MORBIDITY IN PATIENTS WITH \\ CHOLEDOCHOLITHIASIS SUSPICION
}

\author{
Presper F. Daher Filho ${ }^{1}$; Tercio de Campos, TCBC-SP²; Laíse Kuryura ${ }^{3}$; \\ Marcos Belotto ${ }^{3}$; Rodrigo Altenfelder Silva, TCBC-SP ${ }^{4}$; Adhemar M. Pacheco Júnior, TCBC-SP ${ }^{5}$
}

\begin{abstract}
RESUMO: Objetivo: Determinar os fatores preditivos de complicações da colangio-pancreatografia retrógrada endoscópica (CPRE) nos doentes com suspeita diagnóstica de coledocolitíase. Método: Os dados foram coletados retrospectivamente durante o período de agosto de 1999 a janeiro 2005. Foram incluídos os doentes com suspeita diagnóstica de coledocolitíase submetidos à CPRE internados na Santa Casa de Misericórdia de São Paulo. Os doentes com neoplasia de vias biliares ou de pâncreas foram excluídos. Foram avaliados: o sucesso do procedimento, as complicações como pancreatite, sangramento, colangite, perfuração, vômitos, hiperamilasemia e em quais situações estas complicações se desenvolveram. Os testes t de Student, Qui-quadrado e o teste exato de Fisher foram empregados para análise estatística, considerando-se $\mathrm{p}<0,05$ como significativo. Resultados: Cento e setenta oito doentes foram incluídos, sendo 52 homens e 126 mulheres com média etária de 54,3 $\pm 19,3$. A CPRE confirmou o diagnóstico de coledocolitíase em 124 doentes $(69,7 \%)$, obtendo sucesso no tratamento endoscópico em 92 casos $(74,2 \%)$. As complicações foram detectadas em 19 doentes $(10,7 \%)$, com seis casos de pancreatite aguda $(3,4 \%)$, quatro $(2,2 \%)$ que tiveram sangramento durante o procedimento endoscópico, dois $(1,1 \%)$ doentes que desenvolveram colangite, um $(0,6 \%)$ com perfuração duodenal, quatro $(2,2 \%)$ com vômitos sem pancreatite e dois $(1,1 \%)$ com complicações clinicas. A cateterização do ducto pancreático durante a realização do procedimento endoscópico esteve associada com o desenvolvimento de pancreatite aguda ( $\mathrm{p}=0,004)$. Conclusão: A cateterização do pâncreas durante a CPRE constituiu um fator preditivo para o desenvolvimento de pancreatite aguda pós-CPRE (Rev. Col. Bras. Cir. 2007; 34(2): 114-118).
\end{abstract}

Descritores: Coledocolitíase; Pancreatocolangiografia retrógrada endoscópica; Pancreatocolangiografia retrógrada endoscópica/ estatística \& dados numéricos; Pancreatite.

\section{INTRODUÇÃO}

A coledocolitíase é uma condição benigna que ocorre entre 8 e $20 \%$ dos doentes com colelitíase sintomática ${ }^{1-4}$. Este achado leva à necessidade de intervenção na via biliar principal, que poderá ser endoscópica ou cirúrgica ${ }^{5}$.

O tratamento endoscópico pode ser indicado no período pré-operatório, no pós-operatório ou eventualmente durante a colecistectomia ${ }^{2}$. O tratamento cirúrgico é realizado durante a colecistectomia através da exploração pelo ducto cístico ou através da coledocotomia clássica. Recentemente esta exploração das vias biliares tem sido realizada também por videolaparoscopia $(\mathrm{VL})^{6}$.

Quando se estabelece o diagnóstico no período préoperatório, o tratamento endoscópico, através da colangiopancreatografia retrógrada endoscópica (CPRE) com papilotomia e retirada dos cálculos, é o procedimento de escolha para diversos autores, com colecistectomia complemen$\operatorname{tar}^{2,4,6,7}$. Com esta estratégia de tratamento, pode-se simplificar o procedimento operatório ${ }^{8}$. Porém, encontram-se dois obstáculos para que se possa empregar esta tática de tratamento: o primeiro é a determinação do diagnóstico pré-operatório da coledocolitíase; e o segundo são as complicações inerentes à CPRE.

Para o diagnóstico pré-operatório, demonstramos anteriormente que com os atuais métodos disponíveis, como a ecoendoscopia e a colangioressonância, aliados aos dados clínicos e laboratoriais, determina-se a probabilidade de coledocolitíase indicando-se a CPRE para os doentes com maior chance de apresentarem coledocolitíase e que podem ser tratados no mesmo tempo por endoscopia ${ }^{9}$.

Esta proposta evita a realização da CPRE em um número elevado de doentes, reduzindo o custo total do tratamento e o número de complicações do método.

As complicações relacionadas à CPRE estão presentes em cerca de $10 \%$ dos doentes submetidos a este procedimento, sendo a pancreatite aguda responsável por até $50 \%$ delas $^{10}$.

O objetivo do estudo é identificar os fatores relacionados com as complicações após a CPRE em doentes que,

1. Residente do Departamento de Cirurgia da Faculdade de Ciências Médicas da Santa Casa de São Paulo.

2. Pós-Graduando da Faculdade de Ciências Médicas da Santa Casa de São Paulo.

3. Alunos da Faculdade de Ciências Médicas da Santa Casa de São Paulo.

4. Chefe da Disciplina de Vias Biliares e Pâncreas do Departamento de Cirurgia da Faculdade de Ciências Médicas da Santa Casa de São Paulo.

5. Professor Adjunto da Disciplina de Vias Biliares e Pâncreas do Departamento de Cirurgia da Faculdade de Ciências Médicas da Santa Casa de São Paulo.

Recebido em 15/09/2006

Aceito para publicação em 24/11/2006

Conflito de interesses: nenhum

Fonte de financiamento: nenhuma

Trabalho realizado no Grupo de Vias Biliares e Pâncreas do Departamento de Cirurgia da Faculdade de Ciências Médicas da Santa Casa de São Paulo. 
após avaliação de dados clínicos, laboratoriais e de imagem, houve a suspeição de serem portadores de coledocolitíase em associação à litíase vesicular.

\section{MÉTODO}

Trata-se de um estudo retrospectivo baseado no levantamento de feitos entre de agosto de 1999 até janeiro de 2005, nos pacientes tratados pelo Grupo de Vias Biliares e Pâncreas da Faculdade de Ciências Médicas da Santa Casa de São Paulo. Foram incluídos todos os pacientes internados com suspeita de coledocolitíase e submetidos à CPRE.

Para se determinar qual o doente que seria submetido à CPRE foram utilizados critérios clínicos (presença ou antecedente de icterícia ou pancreatite), bioquímicos (elevação de bilirrubinas, fosfatase alcalina ou gamaglutamil-transferase) e ultra-sonográficos (dilatação de vias biliares ou visibilização de cálculo no colédoco). Os doentes com neoplasia de vias biliares ou de pâncreas foram excluídos.

As variáveis estudadas foram: idade, sexo, níveis de leucócitos e bilirrubina, cateterismo do ducto pancreático principal, realização de papilotomia, sucesso do procedimento através da retirada de todos os cálculos e a presença de complicações, como hiperamilasemia assintomática, pancreatite, colangite, perfuração, sangramento, vômitos e outras intercorrências não relacionadas diretamente ao método.

O procedimento endoscópico era realizado sob anestesia geral e consistia na passagem de duodenoscópio de visão lateral, visibilização da papila, seguido de sua cateterização e da injeção do contraste para a realização da colangiografia. Após a revelação do filme os resultados eram interpretados e, caso fosse necessário, o tratamento era realizado durante o mesmo procedimento.

A hiperamilasemia assintomática foi considerada quando o doente apresentava níveis de amilase igual ou acima de três vezes o valor normal, porém sem sintomas clínicos de pancreatite. A pancreatite aguda era definida pela presença de dor abdominal e vômitos por pelo menos 24 horas associada à elevação da amilase acima de três vezes o valor normal. $\mathrm{O}$ diagnóstico de colangite aguda foi baseado na presença de febre persistente por mais de 48 horas do procedimento, além de elevação de enzimas hepáticas.

Os doentes que não desenvolveram complicações eram submetidos à colecistectomia dentro de 24 horas. Caso o procedimento não apresentasse sucesso na resolução da coledocolitíase, o tratamento cirúrgico era indicado na mesma internação dentro de um período de 24 horas. Se o doente evoluísse com algum tipo de complicação a colecistectomia era realizada somente após a total resolução desta complicação.

Como métodos estatísticos foram utilizados o Quiquadrado, o teste exato de Fisher e o teste t de Student, considerando $\mathrm{p}<0,05$ como significativo.

\section{RESULTADOS}

Durante o período de estudo, 178 pacientes foram submetidos à CPRE devido à suspeita diagnóstica de coledocolitíase, sendo 52 homens $(29,2 \%)$ e 126 mulheres (70,8\%). A média etária foi de 54,3 $\pm 19,3$ anos. Um total de $40,4 \%$ dos pacientes apresentava doenças associadas, sendo a hipertensão arterial a mais comum, presente em 48 doentes $(27 \%)$. A CPRE confirmou o diagnóstico de coledocolitíase em 124 doentes $(69,7 \%)$, obtendo sucesso no tratamento endoscópico, ou seja, a retirada de todos os cálculos do colédoco em 92 destes casos (74,2\%). A papilotomia endoscópica foi realizada em 140 doentes $(78,7 \%)$.

As complicações estiveram presentes em 19 doentes $(10,7 \%)$, com seis casos de pancreatite aguda $(3,4 \%)$, quatro $(2,2 \%)$ que tiveram sangramento durante o procedimento endoscópico, dois doentes $(1,1 \%)$ que desenvolveram colangite, um $(0,6 \%)$ com perfuração duodenal, quatro $(2,2 \%)$ com vômitos e dois com complicações clínicas (1.1\%), sendo um com insuficiência respiratória, e outro com taquicardia supra-ventricular (Figura 1). Nove doentes $(5,7 \%)$ tiveram hiperamilasemia assintomática e não foram computados como complicação. Não houve nenhum óbito relacionado ao procedimento endoscópico ou às suas complicações. Todas as complicações foram tratadas clinicamente com sucesso, exceto o caso de perfuração duodenal que foi tratado cirurgicamente e que evoluiu sem intercorrências.

Não houve diferença entre o grupo com complicações e o grupo sem complicações no que se refere à idade, sexo, níveis de leucócitos, nível de bilirrubinas e realização de papilotomia (Tabela 1). Observou-se uma maior incidência no desenvolvimento de pancreatite aguda nos casos em que houve cateterização do ducto pancreático principal.

Do total de 178 doentes, em 165 havia a informação de ocorrência ou não da cateterização do ducto de Wirsung. Dos seis doentes que tiveram a cateterizaçào do ducto de Wirsung, dois $(33,3 \%)$ desenvolveram pancreatite aguda, enquanto que quatro $(2,5 \%)$ dos 159 que não tiveram o ducto pancreático canulado evoluíram com pancreatite aguda ( $\mathrm{p}=$ 0,004). (Figura 2).

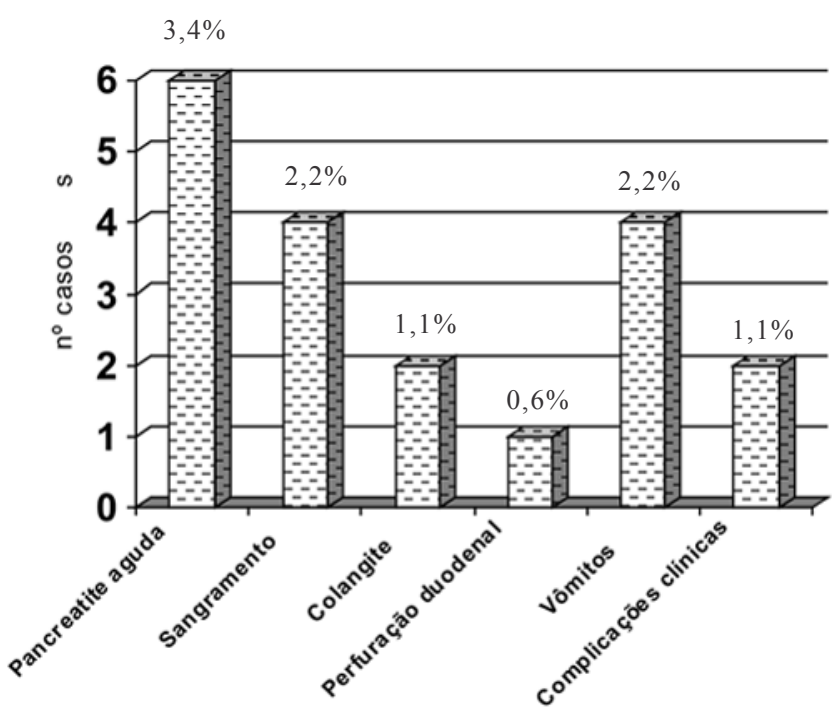

Figura 1 - Complicações relacionadas à CPRE. Total de complicações: 19 casos $(10,7 \%)$ em 178 procedimentos. 
Tabela 1 - Variáveis estudadas comparando o grupo com complicações e o grupo sem complicações.

\begin{tabular}{lccc}
\hline Variável & Com complicações $(\mathbf{n}=\mathbf{1 9})$ & Sem complicações $(\mathbf{n}=\mathbf{1 5 9})$ & $\boldsymbol{p}$ \\
\hline Idade & $56,9 \pm 17$ & $53,9 \pm 19,7$ & 0,526 \\
Sexo feminino & 17 & 103 & 0,056 \\
Leucócitos & $9,879 \pm 5,098$ & $9,347 \pm 5,248$ & 0,676 \\
Bilirrubinas & $8,3 \pm 7,3$ & $5,1 \pm 6,8$ & 0,056 \\
Papilotomia & 5 & 135 & 0,824 \\
\hline
\end{tabular}

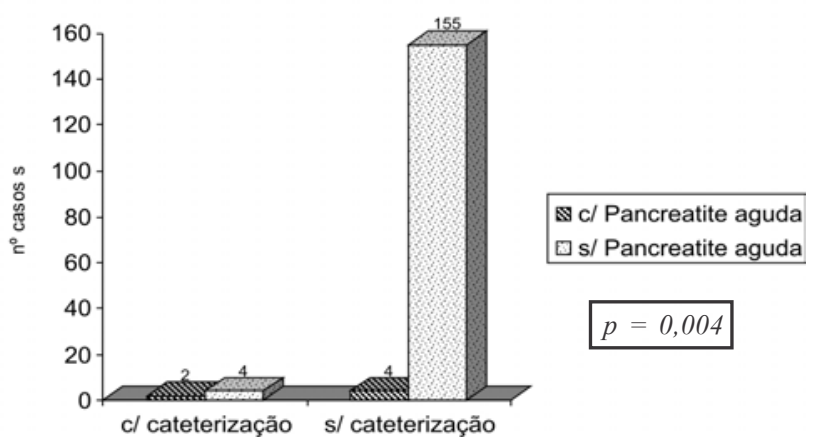

Figura 2 - Relação da cateterização do ducto pancreático com o desenvolvimento de pancreatite aguda (total de casos: 165).

\section{DISCUSSÃO}

As complicações relacionadas à CPRE estão presentes em aproximadamente $10 \%$ dos procedimentos realizados, e podem aumentar caso o procedimento seja terapêutico ${ }^{10-14}$. Deste modo, esta casuística encontrou 19 doentes (10,7\%) com complicações relacionadas à CPRE. Em um levantamento prévio, Parreira et al. não obtiveram complicações relacionadas à CPRE quando analisados doentes com pancreatite aguda biliar ${ }^{13}$.

A complicação mais frequente e mais temida após a realização da CPRE é a pancreatite aguda. A incidência de pancreatite aguda após CPRE varia na literatura de 1\% a 13,5\% e alguns autores relatam a ocorrência de formas graves, com mortalidade de até $27 \%$ destes casos ${ }^{15,16}$. A maior mortalidade na pancreatite aguda grave após a CPRE é atribuído às complicações infecciosas decorrentes de manipulação da região peri-pancreática ${ }^{15}$.

Sugiyama et al. analisaram os fatores de risco para o desenvolvimento de pancreatite aguda após CPRE com dilatação da papila por balão e concluíram que os doentes com pancreatite aguda prévia ou aqueles com via biliar fina apresentaram maior incidência de pancreatite aguda ${ }^{17}$.

Neste estudo, a pancreatite aguda também foi a complicação mais observada, com seis casos $(3,4 \%)$, porém em nenhum dos casos observou-se a forma grave da doença. Detectou-se a ocorrência de pancreatite aguda principalmente nos doentes que tiveram o ducto pancreático cateterizado.

Deve-se ter preocupação com relação ao desenvolvimento de pancreatite aguda nos doentes que serão submetidos à CPRE. Apesar de não haver um consenso, vários estudos têm sido realizados com o intuito de diminuir a chance de uma pancreatite aguda grave após o procedimento endoscópico. A administração de drogas como a somatostatina, o gabexato, a Interleucina 10 e o diclofenaco mostraram evidências de reduzir o risco de pancreatite aguda ${ }^{18,19}$. Porém a medida com melhor efeito parece ser a colocação de stents de pequeno diâmetro (34 Fr) no ducto pancreático ${ }^{20}$.

A canulação traumática da papilla promove freqüentemente um espasmo do esfíncter de Oddi e edema da papila, criando um obstáculo ao fluxo de secreção pancreática e como conseqüência o desenvolvimento de pancreatite aguda ${ }^{21}$. Dessa forma, é provável que este tenha sido o motivo para a presença de pancreatite aguda nos doentes que tiveram a cateterização inadvertida do ducto pancreático. Apesar do pequeno número de casos, esta verificação encontra explicação plausível e é consistente com a literatuta.

Outro dado que merece comentários é o índice de sucesso da extração de cálculos ter sido de $74,2 \%$. Na literatura encontramos índices de limpeza da via biliar que chegam a $99 \%$, quando empregados todos os recursos disponíveis durante o procedimento endoscópico, tais como balões, cestas, litotripsia e várias sessões endoscópicas ${ }^{22,23}$. Porém quando analisamos um único procedimento endoscópico utilizando apenas balão e a cesta em um Serviço de Endoscopia de Hospital Universitário, que é o caso desta casuística, encontramse índices de sucesso de $70-75 \%{ }^{24,25}$.

Portanto, a investigação prévia da probabilidade da presença de coledocolitíase deve ser considerada para indicar-se a CPRE. Em estudo anterior, recomendamos que a CPRE deva ser indicada inicialmente quando um doente apresente icterícia e cálculo visibilizado pela ultra-sonografia9 . Caso contrário, a ecoendoscopia ou a colangioressonância devem ser considerados como opções menos invasivas, para se confirmar o diagnóstico de coledocolitíase e indicar a CPRE com maior precisão ${ }^{26,27}$. Utilizando critérios clínicos, bioquímicos e ultra-sonográficos, indicou-se a CPRE desnecessariamente em 54 doentes $(30,3 \%)$.

A CPRE pode ainda ser utilizada com grande utilidade nos doentes que chegam ao Pronto-Socorro, especialmente em duas situações: a primeira é naqueles com litíase da via biliar complicada com colangite, onde a descompressão endoscópica da via biliar é fundamental. Hui et al. verificaram em doentes com colangite que uma freqüência cardíaca maior que $100 / \mathrm{min}$, albumina menor que $3,0 \mathrm{~g} / \mathrm{dL}$, bilirrubina maior que $50 \mathrm{micromol} / \mathrm{L}$ e tempo de 
protrombina maior que $14 \mathrm{~s}$ na admissão estiveram associados com a necessidade de CPRE de emergência ${ }^{28}$. A segunda situação é a utilização da CPRE nos doentes com pancreatite aguda biliar antes da realização da colecistectomia. Nossa experiência prévia mostrou que $22,4 \%$ dos doentes com pancreatite aguda biliar necessitaram de CPRE previamente à colecistectomia ${ }^{29}$.

Apesar das complicações relatadas, a CPRE também deve ser considerada como uma alternativa à colecistectomia eletiva em doentes com risco cirúrgico elevado devido a doenças associadas.

Concluímos com este estudo que as complicações relacionadas à CPRE são de baixa incidência, mas potencialmente graves e que o único fator relacionado à ocorrência de pancreatite aguda nesta amostra foi a cateterização do ducto pancreático principal. As variáveis sexo, idade, leucocitose, bilirrubinas e realização de papilotomia não estiveram relacionadas com o desenvolvimento de complicações.

\begin{abstract}
Background: To determine the predictors of complications resulting from endoscopic retrograde cholangiopancreatography (ERCP) in patients with choledocholithiasis. Methods: Data were retrospectively collected from August 1999 to January 2005. Patients with clinical diagnosis of choledocholithiasis were included. Patients with tumours of the biliary tree or pancreas were excluded. The authors evaluated the procedure success and the risk factors associated with post-ERCP complications such as acute pancreatitis, hemorrhage, cholangitis, perforation, vomits and hyperamylasemia, and their association with the procedures. Student's $t$ test, the Chi-square and the Fisher exact test were applied to the statistical analysis, considering $p<0.05$ as significant. Results: There were 178 patients $(52$ men and 156 women, mean age $54.3 \pm 19.3)$ included in the analysis. Choledocholithiasis was found in 124 patients (69.7\%) and the sucess rate of the procedure was $74.2 \%$ (92 patients). PostERCP complications occurred in 19 patients (10.7\%). Six patients (3.4\%) had acute pancreatitis, four had hemorrhage from the papilla (2.2\%), two had cholangitis (1.1\%), one had duodenal perforation (0.6\%), and other four had vomiting without pancreatitis $(2.2 \%)$. Two patients had other clinical complications. The main pancreatic duct catheterization was identified as a risk factor for the development of acute pancreatitis after ERCP $(p=0,004)$. Conclusion: Pancreatic duct catheterization during ERCP significantly increases the risk of acute pancreatitis post-ERCP.
\end{abstract}

Key words: Choledocholithiasis; Cholangiopancreatography, endoscopic retrograde; Cholangiopancreatography, endoscopic retrograde/ statistics \& numerical data; Pancreatitis.

\section{REFERÊNCIAS}

1. Tham TCK, Collins JS, Watson RG, Ellis PK, McIlrath EM. Diagnosis of common bile duct stones by intravenous cholangiography: prediction by ultrasound and liver function tests compared with endoscopic retrograde cholangiography. Gastrointest Endosc. 1996; 44(2):158-63.

2. Basso N, Pizzuto G, Surgo D, Matéria A, Silecchia G, Fantini A, Fiocca F, Trentino P. Laparoscopic cholecystectomy and intraoperative endoscopic sphincterotomy in the treatment of cholecysto-choledocholithiasis. Gastrointest Endosc. 1999;50(4):532-5.

3. Liu TH, Consorti ET, Kawashima A, Ernst RD, Black CT, Greger PH Jr, Fischer RP, Mercer DW. The efficacy of magnetic resonance cholangiography for the evaluation of patients with suspected choledocholithiasis before laparoscopic cholecystectomy. Am J Surg. 1999; 178(6):480-4.

4. Menezes N, Marson LP, Debeaux AC, Muir IM, Auld CD. Prospective analysis of a scoring system to predict choledocholithiasis. Br J Surg. 2000; 87(9):1176-81.

5. Suc B, Escat J, Cherqui D, FourtaNIER g, Hay JM, Fingerhut A, Millat B. Surgery vs endoscopy as primary treatment in symptomatic patients with suspected common bile duct stones: a multicenter randomized trial. French Associations for Surgical Research. Arch Surg. 1998;133(7):702-8.

6. Materia A, Pizzuto G, Silecchia G, Fiocca F, Fantini A, Spaziani E, Basso N. Sequential endoscopic-laparoscopic treatment of cholecystocholedocholithiasis. Surg Laparosc Endosc. 1996;6(4):273-7.

7. Hawasli A, Lloyd L, Cacucci B. Management of choledocholithiasis in the era of laparoscopic surgery. Am Surg. 2000;66(5):425-30; discussion 430-1.
8. Rosenthal RJ, Rossi RL, Martin RF. Options and strategies for the management of choledocholithiasis. World J Surg. 1998;22(11):1125-32.

9. De Campos T, Parreira JG, MoriczA, Rego RE, Silva RA, Pacheco Jr AM. Fatores preditivos de coledocolitíase em doentes com litíase vesicular. Rev Assoc Med Bras. 2004;50(2):188-94.

10. Ong TZ, Khor JL, Selamat DS, Yeoh KG, Ho KY. Complications of endoscopic retrograde cholangiography in the post-MRCP era: a tertiary center experience. World J Gastroenterol. 2005;11(33):5209-12.

11. Tanner AR. ERCP: present practice in a single region. Suggested standards for monitoring performance. Eur J Gastroenterol Hepatol. 1996;8(2):145-8.

12. Loperfido S, Angelini G, Benedetti G, Chilovi F, Costan F, De Berardinis F, De Bernardin M, Ederle A, Fina P, Fratton A. Major early complications from diagnostic and therapeutic ERCP: a prospective multicenter study. Gastrointest Endosc. 1998;48(1):1-10.

13. Parreira JG, Rego RE, Campos T, Moreno CH, Pacheco Jr AM, Rasslan S. Fatores preditivos de coledocolitíase em doentes com pancreatite aguda biliar. Rev Assoc Med Bras. 2004;50(4):391-5.

14. Suissa A, Yassin K, Lavy A, Lachter J, Chermech I, Karban , Tamir A, Eliakim R. Outcome and early complications of ERCP: a prospective single center study. Hepatogastroenterology. 2005;52(62):352-5.

15. Pitchumoni CS, Patel NM, Shah P. Factors influencing mortality in acute pancreatitis: can we alter them? J Clin Gastroenterol. 2005;39(9):798-814.

16. Fung AS, Tsiotos GG, Sarr MG. ERCP- induced acute necrotizing pancreatitis: is it a more severe disease. Pancreas. 1997;15(3):217-21. 
17. Sugiyama M, Abe N, Izumisato Y, Masaki T, Mori T, Atomi Y. Risk factors for acute pancreatitis after endoscopic papillary balloon dilation. Hepatogastroenterology. 2003; 50(54):1796-8.

18. Demols A, Deviere J. New frontiers in the pharmacological prevention of post-ERCP pancreatitis: the cytokines. JOP. 2003;4(1):49-57.

19. Murray B, Carter R, Imrie C, Evans S, O'Suilleabhain C. Diclofenac reduces the incidence of acute pancreatitis after endoscopic retrograde cholangiopancreatography. Gastroenterology. 2003;124(7):1786-91.

20. Rashdan A, Fogel EL, McHenry L Jr, Sherman S, Temkit M, Lehman GA. Improved stent characteristics for prophylaxis of post-ERCP pancreatitis. Clin Gastroenterol Hepatol. 2004;2(4):322-9.

21. Testoni PA. Preventing post-ERCP pancreatitis: where are we? JOP. 2003;4(1):22-32.

22. Bergman JJG, Rauws EA, Tijssen JG, Tytgat GN, Huibregtse K. Biliary endoprostheses in elderly patients with endoscopically irretrievable common bile duct stones: report on 117 patients. Gastrointest Endosc. 1995;42(3):195-201.

23. Hamy A, Hennekinne S, Pessaux P, Lada P, Randriamananjo S, Lermite E, Boyer J, Arnaud JP. Endoscopic sphincterotomy prior to laparoscopic cholecystectomy for the treatment of cholelithiasis. Surg Endosc. 2003;17(6):872-5. Epub 2003 Mar 14.

24. Davidson BR, Neoptolemos JP, Carr-Locke DL. Endoscopic sphincterotomy for common bile duct calculi in patients with gallbladder in situ considered unfit for surgery. Gut. 1988;29(1):114-20.

25. Garcia-Cano J. Success rate for complete choledocholithiasis extraction by means of endoscopic retrograde cholangiopancreatography. Surg Endosc. 2004;18(11):1681-2.
26. Sperlongano P, Pisaniello D, Del Viscovo L, De Falco M, Parmeggiani D, Piatto A, Parmeggiani U. Efficacy of magnetic resonance cholangiopancreatography in detecting common bile duct lithiasis: our experience. Chir Ital. 2005;57(5):635-40.

27. Mesihovic R, Vanis N, Husic-Selimovic A, Prohic D, Gribajcevic M, Tanovic H, Beslic S, Smajlovic F, Saray A. Evaluation of the diagnostic accuracy of the endoscopic ultrasonography results in the patients examined in a period of three years. Med Arh. 2005;59(5):299-302.

28. Hui CK, Lai KC, Yuen MF, Ng M, Lai CL, Lam SK. Acute cholangitis - predictive factors for emergency ERCP. Aliment Pharmacol Ther. 2001;15(10):1633-7.

29. Silva RA, Ueda RYY, Rego REC, Pacheco Jr AM, Fava J, Rasslan $\mathrm{S}$. Tratamento cirúrgico postergado da pancreatite aguda biliar. Rev Col Bras Cir. 2000; 27(3):167-72.

Como citar este artigo:

Daher Filho PF, Campos T, Kuryura L, Belotto M, Silva RA, Pacheco Jr AM.

Avaliação de complicações relacionadas à CPRE em doentes com suspeita de coledocolitíase. Rev Col Bras Cir. [periódico na Internet] 2007; 34(2). Disponível em URL: http://www.scielo.br/rcbc

Endereço para correspondência:

Tercio De Campos

Disciplina de Vias Biliares e Pâncreas - F.C.M. Santa Casa - SP

Rua Dr. Cesário Motta Jr. 112

01221-900 - São Paulo - SP

Fone: 11 2176-7271 - FAX: 11 2176-7271

e-mail: tercio@uol.com.br 